\title{
Congenital Giant Right Atrial Aneurysm: Echocardiographic Diagnosis and Surgical Management
}

\author{
Yao Wang, MD, Aijun Liu, MD, Weihua Ye, MD \\ Department of Cardiovascular Surgery, Chinese PLA General Hospital, Beijing, China
}

\section{ABSTRACT}

Congenital right atrial aneurysm is a rare abnormality and may easily be confused with various anomalies, such as pericardial effusion, pericardial cysts, tumors, and Ebstein's anomaly. Patients with right atrial aneurysm may be asymptomatic; but some patients may develop life-threatening complications, such as arrhythmias, congestive heart failure, or pulmonary embolism. Therefore, it is essential for correct diagnosis and appropriate patient management. We report a case of giant right atrial aneurysm in a 7-year-old boy who presented with progressive protrusion of chest. Echocardiography established the definitive diagnosis and surgical resection was successful.

\section{INTRODUCTION}

Congenital right atrial aneurysm is a rare abnormality [Morrow 1968]. It can be detected at any time from fetal to adult life [Chatrath 2001; Moraes 2001]. Patients with right atrial aneurysm may be asymptomatic; but some patients may develop life-threatening complications, such as arrhythmias, congestive heart failure, or pulmonary embolism [Chatrath 2001; Moraes 2001]. Therefore, it is essential for correct diagnosis and appropriate patient management. We report a case of right atrial aneurysm in a 7-year-old boy who presented with progressive protrusion of chest. Echocardiography established the definitive diagnosis and surgical resection was successful.

\section{CASE REPORT}

A 7-year-old male was referred to our institution with a chief complaint of a 6.5-year history of progressive protrusion of chest, according to his mother. He reported no chest pain, fatigue, palpitation, and dyspnea. On chest computed tomography (CT) performed in a district hospital a mediastinal cyst was diagnosed.

The physical examination did not reveal any abnormal findings except for apparent protrusion deformity of the chest. Electrocardiogram showed accelerated junctional escape rhythm, an incomplete right bundle branch block,

Received Fuly 31, 2016; accepted October 30, 2016.

Correspondence: Yao Wang, Department of Cardiovascular Surgery, Chinese PLA General Hospital, 28 Fuxing Rd, Beijing 100853, China; +86-10-66938336; fax: +86-10-66938336 (e-mail: wyecg@163.com). right axis deviation and left ventricular high-voltage. Chest radiography showed an enlarged cardiac silhouette with a prominent right heart border (Figure 1, left), but no definite diagnosis was made. On magnetic resonance imaging (MRI) anterior mediastinal cystic lesion was diagnosed. MRI did not further clarify the diagnosis.

Transthoracic two-dimensional echocardiography revealed a large saccular cavity originating from the right atrial free wall, which was measured approximately $13.0 \times 6.7 \mathrm{~cm}$, and the right atrium and right ventricle were severely compressed by this cavity (Figure 1, middle). Color flow imaging showed an unrestricted blood flow from the right atrium into the saccular cavity through a broad orifice, about $3.4 \mathrm{~cm}$ in diameter. The wall of the saccular cavity was akinetic and no intracavitary thrombus was identified. The tricuspid valve apparatus and the right ventricle were morphologically normal. There was a concomitant patent foramen ovale with bidirectional shunting. Intravenous contrast echocardiography was then performed by means of a bolus intravenous injection of $5 \mathrm{~mL}$ of agitated saline solution via the right antecubital vein. Contrast echocardiography demonstrated uniform opacification


Figure 1. Preoperative chest $\mathrm{X}$-rays and echocardiography. Chest radiography showed an enlarged cardiac silhouette (left); echocardiographic image of right atrial aneurysm compressing the RA and RV (middle). Contrast echocardiography showed uniform opacification of the aneurysm, RA, and RV (right). LA indicates left atrium; LV, left ventricular; $\mathrm{RA}$, right atrium; $\mathrm{RV}$, right ventricular.
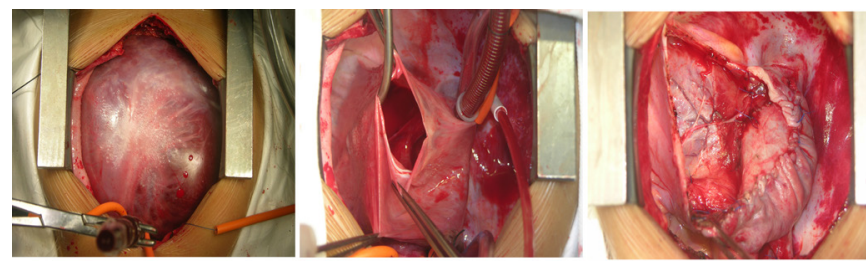

Figure 2. Intraoperative photographs. The right atrial aneurysm occupies almost all pericardial space (left). The wall of the aneurysm was thin and translucent (middle). A pericardial patch was used to close the atrial wall defect (right). 


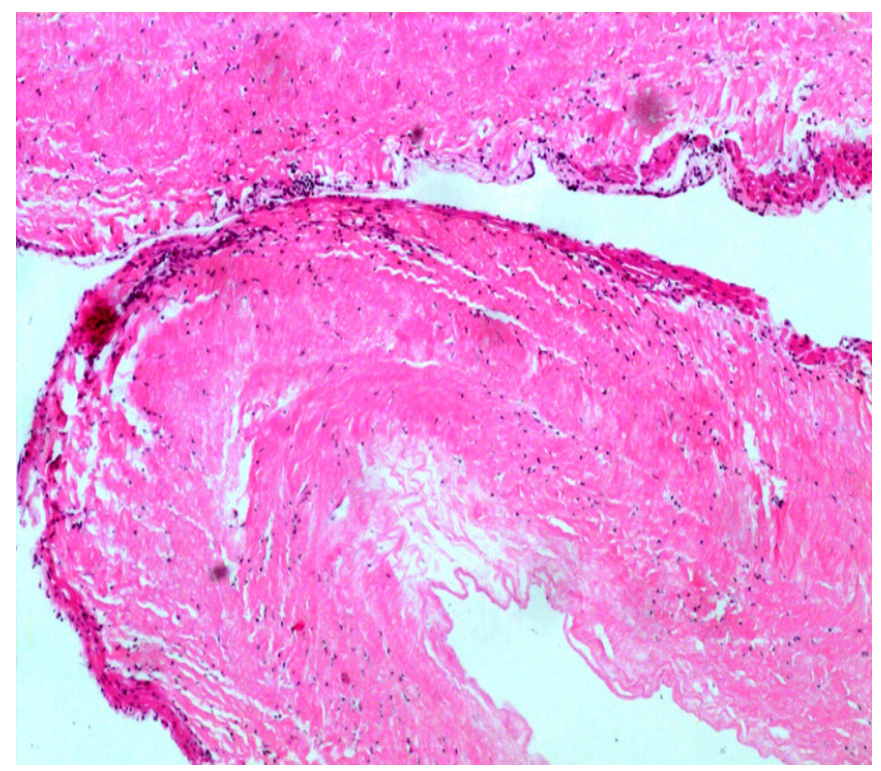

Figure 3. Histology of the wall of the aneurysm. The wall of the aneurysm was composed of fibrous tissue and attenuated myocardial fibers with an endothelial lining.

of the saccular cavity, right atrium, and right ventricle consistent with a congenital giant right atrial aneurysm (Figure 1 , right). The echocardiographic result suggested congenital giant right atrial aneurysm.

The heart was reached through a median sternotomy. Surgical exploration confirmed the echocardiographic findings. A right atrial aneurysm obstructed the whole operating field (Figure 2, left). The wall of the aneurysm was thin and translucent (Figure 2, middle). The aneurysm was excised under cardiopulmonary bypass and a pericardial patch was then used to close the atrial wall defect (Figure 2, right). The patent oval foramen was also closed by direct suturing.

Histologic examination of the specimen revealed that the wall of the aneurysm was composed of fibrous tissue and attenuated myocardial fibers with an endothelial lining (Figure 3).

The postoperative course was eventful. Chest radiograph on the first postoperative day showed a normal sized right atrium and the right ventricle (Figure 4, left). Echocardiography on the 11th postoperative day showed a loculated pericardial effusion $(7.7 \times 4.2 \mathrm{~cm})$ at the lateral wall of the right heart causing localized compression of the right ventricle (Figure 4 , middle). Pericardial fenestration was then performed surgically, and the volume of drainage was about $120 \mathrm{~mL}$. The repeated echocardiography on the fifth day after pericardial fenestration revealed recurrent effusion $(5.1 \times 2.6 \mathrm{~cm})$, with no evidence of cardiac tamponade (Figure 4, right). As the patient was asymptomatic and the clinical condition stable, he was observed without further intervention.

\section{DISCUSSION}

Congenital right atrial aneurysm is a rare heart anomaly. The MEDLINE search revealed that only sporadic cases had

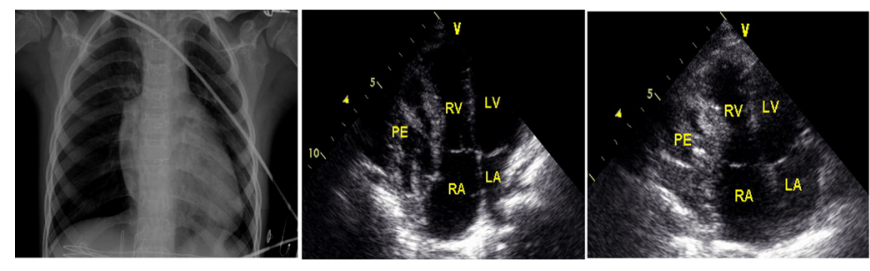

Figure 4. Postoperative chest X-rays and echocardiography. Chest radiography on the first postoperative day showed normal cardiac size after surgery (left). Echocardiography on the 11 th postoperative day showed a loculated pericardial effusion (middle); Echocardiography on the fifth day after pericardial fenestration revealed recurrent effusion (right). LA indicates left atrium; LV, left ventricular; RA, right atrium; $\mathrm{RV}$, right ventricular.

been reported in the literature since the first description by Morrow and Behrendt in 1968 [Morrow 1968]. Congenital right atrial aneurysm can be detected at any time from fetal to late adult life [Chatrath 2001; Moraes 2001]. Some patients were asymptomatic, while others presented with atrial arrhythmias and symptoms associated with it [Chatrath 2001; Moraes 2001]. The most common associated congenital heart defect is an ostium secundum type atrial septal defect [Papagiannis 2008].

Diagnosis and management of congenital right atrial aneurysm are challenging. In respect to the diagnosis of congenital right atrium aneurysm, chest radiography, CT, MRI, and transesophageal echocardiography have been used [Moraes 2001; Imren 2006; Kühn 2006]. It is impossible to confirm such an anomaly with chest radiography even though the condition is suspected because of cardiomegaly on a radiograph. Various anomalies, such as pericardial effusion, pericardial cysts, tumors and Ebstein's anomaly can mimic this malformation on a radiograph [Chatrath 2001]. With CT and MRI, it is difficult to exclude secondary enlargement of the right atrium to enable diagnosis of congenital right atrium malformation. Transesophageal echocardiography is a useful diagnostic tool especially for detection of thrombi inside the aneurysm, but it is an invasive technique and carries a potential risk of aneurysmal rupture and embolization [Imren 2006]. The combination transthoracic echocardiography and contrast echocardiography permit a noninvasive diagnosis of congenital right atrium aneurysm. In the present case, we demonstrated that a combination of transthoracic echocardiography and intravenous agitated saline contrast echocardiography established the definitive diagnosis of right atrial aneurysm.

As far as treatment is concerned, patients with arrhythmias have been treated successfully with excision of the aneurysm [Papagiannis 2008]. In asymptomatic patients, resection of right atrial aneurysm is also recommended due to the potential risk of thrombus formation, arrhythmia, and rupture of the aneurysm [Chatrath 2001; Moraes 2001; Imren 2006]. As in our case, the asymptomatic patient responded well to surgical treatment except for development of postoperative pericardial effusion. The patient was dismissed from the hospital with clinically insignificant pericardial effusion.

Principles of operative management include the use of 
extracorporeal circulation, identification of the communicating orifice, resection of the aneurysm, and then closing the atrial wall defect either by direct suture or use of a pericardial patch to preserve normal atrial size.

In conclusion, congenital right atrium aneurysm can be diagnosed noninvasively by the combination of transthoracic echocardiography and contrast echocardiography. Right atrial aneurysm can be surgically corrected to prevent potential life-threatening complications, such as arrhythmias, congestive heart failure, or pulmonary embolism. The natural course of right atrial aneurysm, however, remains unclear.

\section{REFERENCES}

Chatrath R, Turek O, Quivers ES, Driscoll DJ, Edwards WD, Danielson
GK. 2001. Asymptomatic giant right atrial aneurysm. Tex Heart Inst J 28:301-3.

Imren Y, Halit V, Kula S, Olgunturk R. 2006. Giant right atrial aneurysm: case report. Int J Cardiol 112:e66-8.

Kühn A, Schreiber C, Hausleiter J, Vogt M. 2006. Giant right atrial aneurysm in a symptomatic adult- a rare congenital malformation. Eur Heart J 27:2375.

Moraes F, Santos CL, Lira V, Moraes CR. 2001. Congenital aneurysm of the right atrium. Eur J Cardiothorac Surg 19:943-4.

Morrow AG, Behrendt DM. 1968. Congenital aneurysm (diverticulum) of the right atrium. Clinical manifestations and results of operative treatment. Circulation 38:124-8.

Papagiannis J, Chatzis A, Sarris G. 2008. Giant right atrial aneurysm: a case report. Int J Cardiol 129:e5-6. 